\title{
Estimation of mean sojourn time for lung cancer by chest X-ray screening with a Bayesian approach
}

\author{
Chun-Ru Chien ${ }^{\text {b,d }}$, Mei-Shu Lai ${ }^{a}$, Tony Hsiu-Hsi Chen ${ }^{c, *}$
}

\author{
${ }^{a}$ Institute of Preventive Medicine, College of Public Health, National Taiwan University, Taiwan \\ b Division of Radiation Oncology, Department of Oncology, National Taiwan University Hospital, Taipei, Taiwan \\ c Division of Biostatistics, College of Public Health, National Taiwan University, Taiwan \\ d Department of Radiotherapy and Oncology, China Medical University Hospital, Taichung, Taiwan
}

Received 16 September 2007; received in revised form 10 February 2008; accepted 25 February 2008

\section{KEYWORDS \\ Lung cancer; \\ Chest X-ray; \\ Mean sojourn time; \\ Bayesian approach}

\begin{abstract}
Summary Very few studies, particularly from oriental population, reported the progression of lung cancer from asymptomatic to symptomatic phase. The present study aimed to estimate mean sojourn time (MST) of lung cancer, an average duration period in which tumour can be asymptotically detected by chest X-ray (CXR), taking into account gender, smoking and histological type. Based on institutional cancer registry for lung cancer patients with prior non-diagnostic CXR $(n=221)$, data were collected on demographic features, histology type, survival status, history of smoking, and asymptomatic or symptomatic status in light of chief complaint at diagnosis retrieved from medical records. The MST for the natural history of lung cancer underpinning a three-state Markov model was estimated with a Bayesian approach. The estimated MST for lung cancer was 5.51 months (95\% credible interval: 4.04-7.12). Small cell lung carcinoma was even statistically significantly shorter MST than non-small cell lung carcinoma (3.01 (3-3.98) months vs. 6.07 (4.44-8.25) months). In parallel with literatures reporting tumour growth rate related to CXR and computed tomography (CT), the shorter mean sojourn time by using CXR estimated in our study strongly suggests that CT screening may be more effective in early detection of lung cancer in population-based screening.

(c) 2008 Published by Elsevier Ireland Ltd.
\end{abstract}

\section{Introduction}

Lung cancer is the leading cause of cancer death in the world [1]. The outcomes of lung cancer was dismal, with 5 -year survival around $5 \%$ in the past in Taiwan and around

\footnotetext{
* Corresponding author at: Division of Biostatistics, College of Public Health, National Taiwan University, Room 521, No. 17 Hsuchow Road, Taipei 100, Taiwan. Tel.: +886 2 33228021; fax: +886223587707.

E-mail address: chenlin@ntu.edu.tw (T.H.-H. Chen).
}

$15 \%$ in recent (1995-2001) USA $[2,3]$. To date, there is no standard and acceptable screening method for a person of either normal or high risk for lung cancer, although there are several ongoing trials $[4,5]$. Screening for lung cancer with chest X-ray (CXR) and sputum cytology has been evaluated in several randomized lung cancer screening trials [6-8]. However, there was lacking of evidence on long-term mortality reduction. It is speculated on whether small benefit of screening for lung cancer is attributed to shorter mean sojourn time (MST), namely, average duration of preclinical screen-detectable phase. Studies were few, particularly 
in oriental people, and have been reported to have a wide range between $7-8$ months and 4 years (for early stage disease) $[9,10]$.

Different ethnic/racial groups may have different natural history regarding the progression of lung cancer. In addition to genetic disparity, cigarette smoking was identified as the single most predominant cause of the lung cancer. However, cigarette smoking may only partially explain the epidemiology of lung cancer in Taiwan [2,11,12]. Moreover, the distribution of histological type may also vary from racial group to racial group. Less is known about whether the MST for smoker is shorter than that for non-smoker. Neither is addressed regarding the relationship between histological type like non-small and small cell type and MST. The MST is an important determinant for inter-screening interval and may provide a reference for the feasibility of screening for cancer. In this report, we aimed to estimate the MST of lung cancer early detected by CXR with a Bayesian approach to quantify the natural history of lung cancer by smoking history and histological type in Taiwan based on hospital-based opportunistic screening data collected in a retrospective manner.

\section{Methods}

\subsection{Patients}

From the Institutional Cancer Registry between 1996 and 2002 in the National Taiwan University Hospital, the largest medical center in Taiwan, a retrospective cohort, that is very feasible for opportunistic screening data, was established by enrolling a total of 3201 patients with small cell lung carcinoma (SCLC) and non-small cell lung carcinoma (NSCLC) cases with known surveillance epidemiology and end results (SEER) stage status [13]. To estimate the mean sojourn time for patients early detected by CXR, we extracted 330 patients with prior CXR. To only include patients with prior non-diagnostic (with subsequent workup) CXR, we excluded the subjects with the following criteria: those too old (age at diagnosis $\geq 80$-year-old) $(n=46)$, too young (age at diagnosis $<40$-year-old) $(n=5)$, short interval between diagnosis and prior chest $X$-ray $(n=6)$ (less than 1 year except 6 months for prior chest $X$-ray taken on the grounds of health exam or admission in internal medicine ward), long interval between diagnosis and prior chest X-ray (more than 5 years) $(n=49)$, and suspected missing diagnosis (false negative cases) after checking medical record and X-ray $(n=3)$. The total of 221 patients aged 40-79 years were enrolled for the following analysis of MST. We also compared 221 patients with the remaining 2659 lung cancer patients aged 40-79 years in the contemporaneous period to assess the comparability in respect of demographic features between both groups.

Data on gender, age at diagnosis, history of smoking (ever or never), histological type (NSCLC or SCLC), chief complaint at diagnosis (asymptomatic or symptomatic), and survival status were retrieved from the medical records retrospectively and from the cancer registry system.

\subsection{Model specification}

We modeled the natural history of lung cancer using a three-state Markov model using a Bayesian approach implemented with WinBUGS program [14]. The disease process was depicted in Fig. 1 which has been used in modeling the disease natural history of breast cancer [15]. State 1 represents no disease, state 2 asymptomatic chest X-ray detectable lung cancer and state 3 clinical symptomatic lung cancer. Subjects are assumed to be in state 15 years before diagnosis. If lung cancer is diagnosed asymptomatically, the subject is assumed to have moved to state 2 since the last chest X-ray or in the last 5 years if this is the subject's first $X$-ray. If the subject is diagnosed as a result of symptoms, the subject is deemed to have moved into state 2 since last clear X-ray or since 5 years before, and to have moved to state 3 on the date of diagnosis. The flow chart of disease status by time horizon of these patients $(n=221)$ receiving opportunistic screening is shown in Fig. 2, which is composed of asymptomatic $(n=43)$ or symptomatic $(n=178)$ cases. We used 3 months as an interval unit pursuant to surveillance guidelines [16]. For example, if a patient was diagnosed asymptomatically with most recent non-diagnostic chest Xray taken 1 year ago, the status coding $\left(\mathrm{s}_{0}, \mathrm{~s}_{1}, \ldots, \mathrm{s}_{21}\right)$ would be $1, \ldots, 1$, NA (not available), NA, NA, 2. For another patient diagnosed symptomatically with most recent non-diagnostic chest X-ray taken 5 years ago, the coding would be 1, NA,..., NA, 3. Let $P_{11}$ denote the transition probability from state 1 to state 1 in a 3-month interval. $P_{12}, P_{22}$, and $P_{23}$ was defined in a similar manner. The transition probability from state 1 to state 3 in a 3-month interval was assumed to be very small and negligible, i.e., $P_{13}=0$, in light of the published guideline in which 6 months' surveillance interval after curative therapy was recommended [16]. We assumed that $P_{11}, P_{12}$, $P_{22}$, and $P_{23}$ were time-homogenous (independent of age).

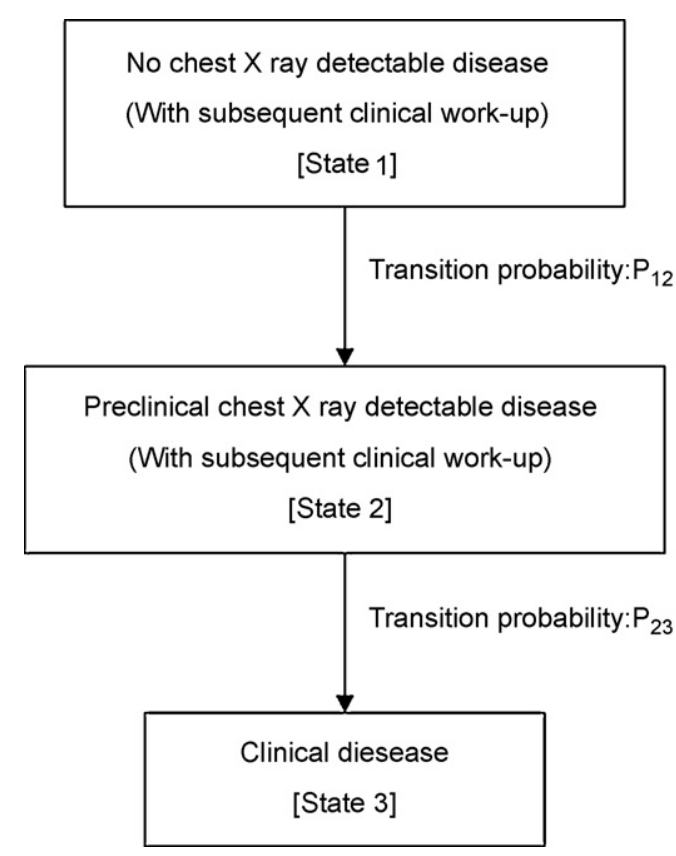

Fig. 1 Three-state Markov model for the disease natural history of lung cancer detected by chest X-ray. 


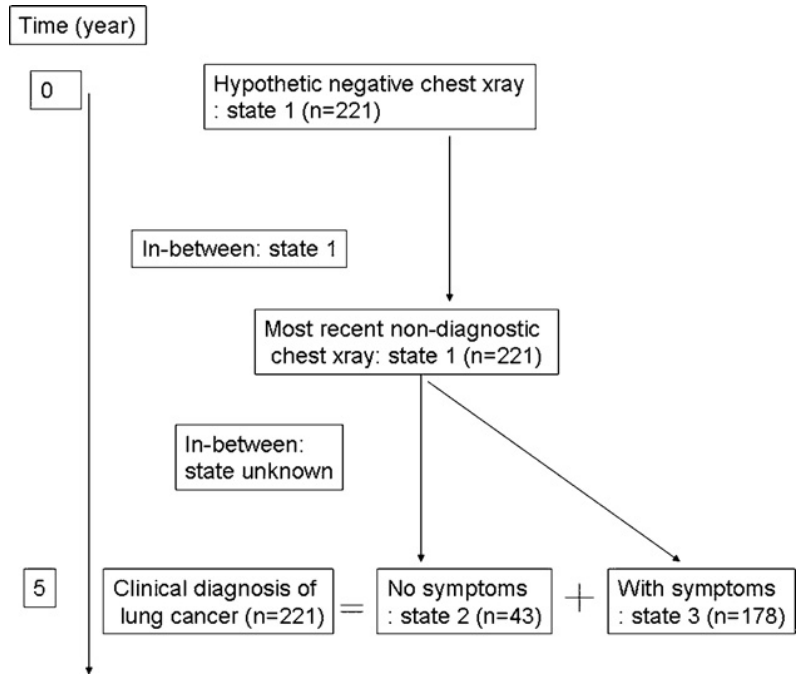

Fig. 2 Disease status of the study population by time horizon.

In order to investigate the effect of gender, smoking, or histology on the transition probabilities, a log linear model was used. The model form is described as follows:

$\log \left(\right.$ relative probability $\left._{i j}\right)$

$$
=\text { Beta }_{i j} \times \text { factor(gender, ..., etc.) }
$$

where $i$ and $j$ are departing and destination states, respectively.

We used vague prior (normal distribution with mean $=0$ and variance $=100$ ) for Beta $_{12}$ and Beta $\mathrm{B}_{23}$. Posterior distributions, from which we drew inferences on transition probabilities, were derived from 5000 iterations. From the 5000 samples, we used the median value as an overall summary data and 2.5th and 97.5th percentiles as the lower- and upper-bounds of the $95 \%$ credible intervals. MST (in month) was approximately estimated by the following equation:

MST $=3 \times \sum_{i=0}^{20} P_{22}^{i}$

The details of approximation are given in Appendix A.

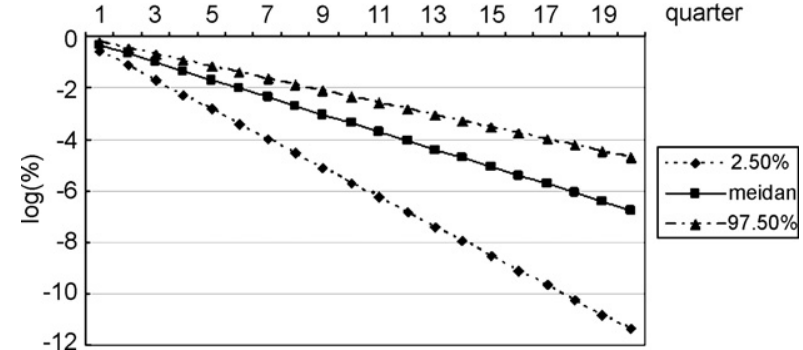

Fig. 3 The logarithm of the probability of retaining in state 2 .

\section{Results}

\subsection{Comparison of the demographic and survival features}

The baseline characteristics of participating group and nonparticipating group are shown in Table 1 . The gender and histology distributions were similar between the study group and the remaining patients. However, the study group had statistically more early stage $(p<0.0024)$ patients and were slightly older than the remaining patients $(p<0.0001)$.

\subsection{The overall MST}

The median and $95 \%$ credible interval $(\mathrm{Cl})$ of MST in general was estimated as 5.51 (4.04-7.12) months. This implied the probability of staying in pre-clinical state for 2 years is around $0.2 \%$ (Fig. 3).

\subsection{Effect of gender, smoking, and histology}

The median and $95 \% \mathrm{Cl}$ of MST (in month) for female versus male, non-smoker versus ever smoker, and NSCLC versus SCLC were 6.7 (4.12-10.20) versus 4.47 (3.00-6.74), 6.72 $(4.32-10.03)$ versus 3.33 (3-6.07), and 6.07 (4.44-8.25) versus $3.01(3-3.98)$, respectively (Table 2$)$. The MST of male, or smoker were shorter compared with female or non-

Table 1 Patient characteristics

\begin{tabular}{|c|c|c|c|}
\hline & Study group $(n=221)$ & The remaining $(n=2659)$ & $p$-Value \\
\hline \multicolumn{4}{|l|}{ Sex } \\
\hline Male & 146 & 1668 & \multirow[t]{2}{*}{0.3241} \\
\hline Female & 75 & 991 & \\
\hline \multicolumn{4}{|l|}{ Stage } \\
\hline Early & 113 & 1081 & \multirow[t]{2}{*}{0.0024} \\
\hline Late & 108 & 1578 & \\
\hline Age (years): mean & 66.4 & 63.5 & $<0.001$ \\
\hline \multicolumn{4}{|l|}{ Histology } \\
\hline SCLC & 20 & 217 & \multirow[t]{2}{*}{0.644} \\
\hline NSCLC & 201 & 2442 & \\
\hline 5-year overall survival & 0.19 & 0.09 & 0.0035 \\
\hline
\end{tabular}

Early: surveillance epidemiology and end results (SEER) stage 2-5; late: SEER stage 7; SCLC: small cell lung cancer; NSCLC: non-small cell lung cancer. 
Table 2 Optimistic estimation of MST of lung cancer

\begin{tabular}{ll}
\hline Subgroup & Median MST $(95 \%$ credible interval) \\
\hline Overall study group & $5.51(4.04-7.12)$ \\
Gender & \\
$\quad$ Female & $6.70(4.12-10.20)$ \\
$\quad$ Male & $4.47(3.00-6.74)$ \\
Smoking & \\
$\quad$ Never & $6.72(4.32-10.03)$ \\
Ever & $3.33(3.00-6.07)$ \\
Histology & \\
$\quad$ Non-small cell & $6.07(4.44-8.25)$ \\
Small cell & $3.01(3.00-3.98)$ \\
\hline
\end{tabular}

smoker but the difference was not statistically significant whereas the difference of MST with respect to histological type was statistically significant, being shorter in SCLC as compared with NSCLC.

\subsection{Subgroup MST}

Due to the sparse cases for the 20 SCLC patients (only 1 female ever-smoker and only 1 male non-smoker), adjustment was made for gender and smoking status only. The estimated median $(95 \% \mathrm{Cl})$ of MST (months) for different subgroups were 3.02 (3-5.68), 4.28 (3-11.79), 3.41 (3-12.89), and $6.73(3-11)$ for male smoker, male non-smoker, female smoker ( $n=11$ only), and female non-smoker, respectively.

\subsection{Model validation}

$\chi^{2}$-Test of goodness-of-fit was used for model validation. We used a hypothetic dataset (all the in-between status were not available (NA) in contrast to only some were NA in our final calculation) for the comparison of the predicted status distribution and the observed status distribution (43 in status 2 and 178 in status 3 ) at time-20 (5th year). The median of $\chi^{2}$ statistic were $1.13(95 \% \mathrm{Cl}: 0-10.8)$. This statistic in most of the time (probability: 0.91, Bayes factor: 10.1 in favor of no difference) was less than $5.99\left(\chi_{2,0.95}^{2}\right)$.

\section{Discussion}

Our report revealed that the MST for lung cancer detected by CXR might be around 6 months at most using a retrospective cohort in Taiwan. Female, non-smoker, and particularly NSCLC tended to have longer MST. MST was one of the key elements of screening program [17]. The outcome was more favorable in the study group than in the remaining patients. The above implies that the MST of our study group may possibly be the optimistic estimation. As our MST estimation may be too short, conventional CXR screening program may not be effective in early detection of lung cancer for Taiwanese population as already reported by Western studies $[4,5]$.

There were few reports focused on the natural history of lung cancer. Based on periodic sputum cytological exam of over 50,000 samples from about 6000 men, Saccomanno et al. estimated that the average period from markedly atypical cells or carcinoma in situ to invasive carcinoma (epidermoid or small cell) was around 4-5 years [18]. Based on mathematical model, Geddes estimated that the average time from earliest diagnosis (assumed to be $1 \mathrm{~cm}$ in size) was around 8.4 years and the average time from malignant change to diagnosis (assumed to be $3 \mathrm{~cm}$ in size of tumour) was about 10 years. The combination of these two finding suggests 1.6 years of MST [19]. But longer (4 years for early stage NSCLC) or shorter (7-8 months) MSTs have also been reported $[9,10]$. Our estimate of MST is close to that of $7-8$ months reported by Walter et al. based on Czech trial [10]. Our MST estimate cannot be directly compared with the mean duration of at least 4 years in stage 1 non-small cell lung cancer reported from Mayo lung project [9] because our estimate is targeted to the overall undetectable tumour whereas the reported estimate of Mayo lung project is limited to average dwelling time of stage 1 non-small cell lung cancer. To be comparable, stage-specific MST should be elucidated in the future. One explanation for short MST is that CXR may detect the majority of asymptomatic lung cancers on the verge of clinical phase.

There were also some literatures estimating the potential effectiveness of screening CXR or CT based on doubling time estimation (for example, Bach et al. [20]). In this review, the doubling time of lung cancer was estimated to be an average of 55 (range: $40-70$ ) days [20]. The required time for the development of clinical lung cancer was estimated as 35 doubling $[19,20]$, whereas the corresponding for chest X-ray detectable tumour was estimated to be 30 doubling [19]. In view that the CXR finding obtained from our study was based on opportunistic screening (usual clinical care) instead of population-based (mass) screening, we assumed the doubling required in this scenario was slightly greater than 30 , say 32 . Therefore, 3 presumptive doubling was required for lung tumour in our study to progress from undetectable in usual care chest X-ray to clinical disease and these imply $165(55 \times 3)$ days of mean sojourn time, which was close to our MST (median 168 days, 95\% Cl: 123-217 days).

The cases selection may be biased toward the better prognosis of our study group. However, this would make our estimation of MST to be relative optimistic. Given so optimistic cases, the MST estimation was short, so CXR may not be effective as a screening modality for lung cancer. On the other hand, since cases were selected from a tertiary medical center, the real MST for general population may be better than our estimation. However, based on our narrow $95 \% \mathrm{Cl}$ (mostly less than 13 months, even for favorable subgroup), this may be unlikely.

Since retrieving information on relevant variables was retrospective in our study, the validity of our estimated results may be argued. However, since the exclusion criteria like age and interval since last CXR is rather explicit and biologically reasonable and, besides, our estimation was comparable to results estimated from prospective randomized screening trial [10], the accuracy of estimated results is reliable and valuable. We excluded those younger than 40 years or older than 80 years in order to make our result feasible and cost-effective for screening program. Those younger than 40 years or older than 80 years is unlikely to be invited into screening due to the low yield for those younger than 40 
years (the incidence, particularly in developing country, is low) and little gain of life years for those older than 80 years which is not cost-effective. Among the studies included in a systematic review [21], the eligibility of age range for screening trials was between 40 and 85 years old, which is very similar to our inclusion criteria.

In addition, since the estimation of MST is based on a mathematical model that may require several assumptions. First, the definition of "non-diagnostic" CXRs assume all these patients had undetectable lung cancer. This assumption seems reasonable in that the exclusion of subjects with short interval as mentioned in Section 2 was to exclude false negative cases assuming 6 months or 1 year is the upper limit of time to find interval cancer surfacing to clinical phase. Because of this exclusion [22], it is reasonably to assume $100 \%$ sensitivity in the estimation of mean sojourn time. On the other hand, we excluded those with prior CXR taken 5 years ago since it is unlikely to have sojourn time for lung cancer greater than 5 years based on published literatures $[9,10]$. It is reasoned that the effect of the finding of CXR taken 5 years or longer ago may be probably unrelated to the potential subsequent screening. If it is related, the probable one is over-diagnosis. To exclude those with prior CXR taken 5 years ago may also reduce such a bias. When we changed the assumption of being in state 1 for 6 years before diagnosis instead of 5 years, the median estimated MST was 5.27 months (95\% Cl: 4.15-6.39), which is close to our base-case estimate $(5.51,95 \% \mathrm{Cl}$ : 4.04-7.12). Second, the transition probability from undetectable to clinically symptomatic lung cancer denoted by $P_{13}$ ( $t=3$ months) was assumed to be zero based on published guideline $[16,23]$, in which 6 months' interval was suggested as surveillance interval after curative therapy. Note that $P_{13}(t=3$ months) is roughly equal to one-fourth of annual incidence. Even for high-risk population included in lung cancer screening trial [24], $P_{13}$ ( $t=3$ months) will be around 0.001 , which is the almost upper bound of this estimate. In our sensitivity analysis, the upper bound of $P_{13}$ is taken as $0.01,10$-fold of high-risk group estimate, the corresponding median (95\% $\mathrm{Cl})$ MST (month) was 5.79 (4.26-7.73), which is not different from our base-case estimate $(5.51,95 \% \mathrm{Cl}$ : $4.04-7.12)$ assuming $P_{13}$ as zero. Therefore, it seems reasonable to assume $P_{13}$ ( $t=3$ months) as zero for our estimation.

Another concern is that our estimation of MST was based on preclinical CXR (and subsequent work-up) detectable phase. It may be different if other modalities were adopted (for example, computed tomography (CT) in some ongoing clinical trials) [5]. Although there is still ongoing chest Xray screening trial [25], the most promising modality for lung cancer screening now is the CT [20]. To our knowledge, no published data was available regarding the MST of lung cancer by screening CT. Based on similar approach mentioned above and the doubling required for $\mathrm{CT}$ detectable lung cancer was, on average 25 (range: 22-28) [20], and 10 doubling was required for progression into clinical disease. Then, the estimated MST would be around $550(55 \times 10)$ days, which suggests CT screening is more effective in early detection of lung cancer compared with X-ray screening. Besides, the trends we found in subgroups (longer MST in female, non-smoker, and NSCLC) may also be of value in our understanding of the natural history of lung cancer and future design of CT screening trial.

\section{Conclusion}

The MST of lung cancer in Taiwan was estimated as 6 months, ranging from 4 to 7 months. Small cell lung carcinoma had even shorter MST. The shorter sojourn time renders the screening tool with CXR difficult to have early detection of lung cancer from the underlying general population. In parallel with the literatures on the growth rates of tumour related to doubling time detected by CXR and CT, our result may not only suggest that CT screening is more effective in early detection of lung cancer than CXR and also provide a supplemental evidence for the future design and potential success of CT screening for lung cancer.

\section{Conflict of interest}

The authors declared no conflict of interest.

\section{Appendix A}

In the light of the definition of the expected estimate, the mean estimate of sojourn time was

$$
\int_{0}^{\infty} t \lambda \exp (-\lambda t) d t
$$

By using integration by part, the equation above was equal to

$$
\int_{0}^{\infty} \exp (-\lambda t) d t
$$

Since the sojourn time longer than 5 years for lung cancer is very unlikely, the infinity of upper limit is replaced by 20 periods (60 months) and the equation can be approximated by

$$
\sum_{n=20}^{20}\left[\exp (-\lambda 3)^{n} \times 3\right]=3 \sum_{n=0}^{20} P_{22}^{n} \quad(\because \text { interval }=3 \text { months })
$$

\section{References}

[1] WHO's World Health Statistics [database on the Internet]. Global Burden of Disease Estimates: 2004 revision of the World Health Report; 2002 [cited September 15, 2007]. Available from: http://www.who.int/healthinfo/statistics/gbdwhoregi onmortality2002.xls.

[2] Luh KT, Chang DB. Primary lung cancer in Taiwan. J Formos Med Assoc 1992;91(Suppl 1):S1-7.

[3] National Cancer Institute [homepage on the Internet]. Surveillance epidemiology and end results: cancer of the lung and bronchus [cited September 15, 2007]. Available from: http://seer.cancer.gov/statfacts/html/lungb.html?statfacts _page=lungb.html\&x=15 \&y=18.

[4] Brawley OW, Kramer BS. Cancer screening in theory and in practice. J Clin Oncol 2005;23:293-300.

[5] Mulshine JL, Sullivan DC. Lung cancer screening. N Engl J Med 2005;352:2714-20.

[6] Levin ML, Tockman MS, Frost JK, Ball Jr WC. Lung cancer mortality in males screened by chest X-ray and cytologic sputum 
examination: a preliminary report. Recent Results Cancer Res 1982;82:138-46.

[7] Fontana RS. The Mayo lung project: a perspective. Cancer 2000;89:2352-5.

[8] Melamed MR, Flehinger BJ, Zaman MB, Heelan RT, Perchick WA, Martini N. Screening for early lung cancer. Results of the Memorial Sloan-Kettering study in New York. Chest 1984;86: 44-53.

[9] Flehinger BJ, Kimmel M, Polyak T, Melamed MR. Screening for lung cancer. The Mayo lung project revisited. Cancer 1993;72:1573-80.

[10] Walter SD, Kubik A, Parkin DM, Reissigova J, Adamec M, Khlat $M$. The natural history of lung cancer estimated from the results of a randomized trial of screening. Cancer Causes Cont 1992;3:115-23.

[11] Liaw YP, Huang YC, Lien GW. Patterns of lung cancer mortality in 23 countries: application of the age-period-cohort model. BMC Publ Health 2005;5:22.

[12] Alberg AJ, Brock MV, Samet JM. Epidemiology of lung cancer: looking to the future. J Clin Oncol 2005;23:3175-85.

[13] Young Jr JL, Roffers SD, Ries LAG, Fritz AG, Hurlbut AA, editors. SEER summary staging manual-2000: codes and coding instructions [monograph on the Internet] NIH Pub. No. 014969. Bethesda, MD: National Cancer Institute; 2001 [cited November 1, 2006]. Available from: http://seer.cancer.gov/ tools/ssm.

[14] The BUGS Project [homepage on the Internet]. Cambridge, UK: MRC Biostatistics Unit; 1996-2004 [cited December 26, 2005]. Available from: http://www.mrc-bsu.cam.ac. uk/bugs.

[15] Chen TH, Kuo HS, Yen MF, Lai MS, Tabar L, Duffy SW. Estimation of sojourn time in chronic disease screening without data on interval cases. Biometrics 2000;56:167-72.
[16] Colice GL, Rubins J, Unger M. Follow-up and surveillance of the lung cancer patient following curative-intent therapy. Chest 2003;123:272S-83S.

[17] Shen Y, Zelen M. Screening sensitivity and sojourn time from breast cancer early detection clinical trials: mammograms and physical examinations. J Clin Oncol 2001;19:3490-9.

[18] Saccomanno G, Archer VE, Auerbach O, Saunders RP, Brennan LM. Development of carcinoma of the lung as reflected in exfoliated cells. Cancer 1974;33:256-70.

[19] Geddes DM. The natural history of lung cancer: a review based on rates of tumour growth. Br J Dis Chest 1979;73:1-17.

[20] Bach PB, Silvestri GA, Hanger M, Jett JR. Screening for lung cancer: ACCP evidence-based clinical practice guidelines (2nd edition). Chest 2007;132:69S-77S.

[21] Yau G, Lock M, Rodrigues G. Systematic review of baseline lowdose CT lung cancer screening. Lung Cancer 2007;58:161-70.

[22] Day NE. Estimating the sensitivity of a screening test. J Epidemiol Community Health 1985;39:364-6.

[23] Rubins J, Unger M, Colice GL. Follow-up and surveillance of the lung cancer patient following curative intent therapy: ACCP evidence-based clinical practice guideline (2nd edition). Chest 2007;132:355S-67S.

[24] van lersel CA, de Koning HJ, Draisma G, Mali WP, Scholten ET, Nackaerts $K$, et al. Risk-based selection from the general population in a screening trial: selection criteria, recruitment and power for the Dutch-Belgian randomised lung cancer multi-slice CT screening trial (NELSON). Int J Cancer 2007;120:868-74.

[25] Andriole GL, Levin DL, Crawford ED, Gelmann EP, Pinsky PF, Chia D, et al. Prostate cancer screening in the prostate, lung, colorectal and ovarian (PLCO) cancer screening trial: findings from the initial screening round of a randomized trial. J Natl Cancer Inst 2005;97:433-8. 